\title{
Effects of local anesthetic or systemic analgesia on pain associated with cautery disbudding in calves: A systematic review and meta-analysis
}

\author{
Charlotte B. Winder, ${ }^{* 1}$ Cynthia L. Miltenburg, ${ }^{*}$ Jan M. Sargeant, ${ }^{*} \dagger$ Stephen J. LeBlanc, ${ }^{*}$ Derek B. Haley, \\ Kerry D. Lissemore, ${ }^{*}$ M. Ann Godkin, $\ddagger$ and Todd F. Duffield* \\ *Department of Population Medicine, and \\ †Centre for Public Health and Zoonoses, University of Guelph, Ontario, N1G 2W1 Canada \\ †Ontario Ministry of Agriculture, Food, and Rural Affairs, Elora, Ontario, NOB 1S0 Canada
}

\begin{abstract}
Disbudding is a common management procedure performed on dairy farms and, when done without pain mitigation, is viewed as a key welfare issue. Use of pain control has increased in recent years, but full adoption of anesthesia and analgesia by veterinarians or dairy producers has not been achieved. This may in part be due to the lack of a consistent recommendations of treatment protocols between studies examining pain control methods for disbudding. The objective of this systematic review was to examine the effects of these pain control practices for the most common method of disbudding, cautery, on outcomes associated with disbudding pain in calves. The outcomes were plasma cortisol concentrations, pressure sensitivity of the horn bud area, and validated pain behaviors (ear flick, head shake, head rub, foot stamp, and vocalization). Intervention studies describing cautery disbudding in calves $12 \mathrm{wk}$ of age or younger were eligible, provided they compared local anesthesia, nonsteroidal anti-inflammatory drug (NSAID), or local anesthesia and NSAID to 1 or more of local anesthesia, NSAID, or no pain control. The search strategy used the Agricola, Medline (via OvidSP), and Web of Science databases, as well as the Searchable Proceedings of Animal Conferences (S-PAC), ProQuest Dissertations and Theses Database, and Open Access Theses and Dissertations. Meta-analysis was performed for all outcomes measured at similar time points with more than 2 studies. Local anesthetic was associated with reduced plasma cortisol until $2 \mathrm{~h}$ postdisbudding; however, a rise in cortisol was observed in the meta-analysis of studies reporting at 4 $\mathrm{h}$ postdisbudding. Heterogeneity was present in several of the analyses for this comparison. The addition of NSAID to local anesthetic showed reduction in plasma
\end{abstract}

Received November 2, 2017.

Accepted January 20, 2018.

${ }^{1}$ Corresponding author: winderc@uoguelph.ca cortisol at $4 \mathrm{~h}$, and a reduction in pressure sensitivity and pain behaviors in some analyses between 3 and 6 $\mathrm{h}$ postdisbudding. Heterogeneity was present in some meta-analyses, including several using pain behavior outcomes. This may reflect the variation in measurement time periods for behavioral measures between studies, as well as differences among NSAID treatments. Overall, a protective effect of local anesthetic was seen for the acute pain of cautery disbudding, and the delayed rise in cortisol was mitigated by the addition of an NSAID, which also reduced other signs of pain, including pressure sensitivity and pain behaviors. Based on these findings, we recommend use of local anesthetic and an NSAID as best practices for pain mitigation for cautery disbudding of calves $12 \mathrm{wk}$ of age or less. The magnitude and duration of the effect of NSAID treatment was not possible to deduce from the literature because wide variation existed between studies. We recommend consideration of more standardized outcome measurements, especially for pain behaviors. Adherence to reporting guidelines by authors would help ensure more transparent and complete information is available to end users.

Key words: systematic review, meta-analysis, disbudding, pain

\section{INTRODUCTION}

Pain control for the disbudding or dehorning of cattle is a key animal welfare issue in the dairy industry (Ventura et al., 2015). Although NSAID analgesia in addition to local anesthesia has generally been found to be beneficial, the lack of specific recommendations for analgesia protocols may reflect the variety examined in the literature. Full compliance has not been achieved by producers or veterinarians in North America with regard to the use of local anesthetic and a nonsteroidal anti-inflammatory drug (NSAID; Adams et al., 2015; Winder et al., 2016), which is the current recommendation of industry and veterinary groups regarding pain 
control (Canadian Veterinary Medical Association, 2010; American Veterinary Medical Association, 2014; Dairy Farmers of Canada, 2015). Although arresting horn growth can be done by surgical amputation, cautery, or use of chemical methods, cautery disbudding remains the most commonly used method by dairy producers in North America, with 89, 70, and 77\% reporting use in the United States; Ontario, Canada; and Quebec, Canada, respectively (Vasseur et al., 2010; Adams et al., 2015; Winder et al., 2016).

Part of the gap between primary research and application in the dairy industry may be driven by the lack of a consistent set of recommendations from primary research papers. Likewise, narrative reviews typically do not include evidence-based methods to identify, assess, and synthesize results; as a result, conclusions may suffer from bias. Conversely, properly conducted systematic reviews offer a more robust and transparent methodology to identify, evaluate, and summarize evidence on a given topic (Sargeant and O'Connor, 2014). Meta-analyses also allow for synthesis of overall effects as well as identification and exploration of causes of heterogeneity among studies, possibly identifying sources of variability that may be further examined or used to guide inferences of the robustness of the observed effects across different study designs or settings (Sargeant and O'Connor, 2014).

The objectives of our systematic review were to examine the effects of local anesthesia or NSAID analgesia on plasma cortisol, pressure sensitivity, and pain behaviors following cautery disbudding. If enough studies reported on a given outcome at a similar time point, meta-analysis was conducted. This review should serve as a stronger form of evidence for the effects of these practices than narrative reviews or the results of a single research study. Our review will identify gaps in this body of literature and the degree, or lack, of homogeneity among reported interventions and outcomes, which should serve to inform future research designs and study reporting. This manuscript was prepared in accordance with Preferred Reporting Items for Systematic Reviews and Meta-Analyses: The PRISMA statement (Moher et al., 2010).

\section{MATERIALS AND METHODS}

\section{Protocol and Registration}

A review protocol was created a priori in accordance with PRISMA-P guidelines (Moher et al., 2015) and deposited with the University of Guelph Atrium on April 26, 2017 (http://hdl.handle.net/10214/10324) and is also available via Systematic Reviews for Ani- mals and Food (http://www.syreaf.org/contact/). The protocol is included in Supplemental File S1 (https:// doi.org/10/3168/jds.2017-14092).

\section{Eligibility Criteria}

Primary Study Design, Characteristics, and Populations. Primary experimental intervention studies available in English, including both randomized and nonrandomized clinical trials, were eligible for inclusion. Observational study designs were not eligible. Studies must have involved bovine calves 12 wk of age or less who underwent cautery disbudding with no concurrent painful procedures, defined as 1 or more of castration, branding, or any surgical procedure.

Intervention and Comparator Groups. Eligible studies must have included at least 2 of the following experimental groups: no pain control given, local anesthetic alone, NSAID alone, or local anesthetic and NSAID.

Outcome Measures. Many outcomes have been used in disbudding studies as indicators of pain. For inclusion in our systematic review, studies must have included 1 or more of the following outcomes, measured at 1 or more time points: plasma cortisol, pain behaviors (one or more of ear flick, head shake, head rub, tail swish, foot stamp, and vocalization; Faulkner and Weary, 2000; Heinrich et al., 2010), or sensitivity of the horn bud (e.g., measured by an algometer or von Frey monofilaments; Heinrich et al., 2010; Mintline et al., 2013). These outcomes were chosen based on consideration of their use in the literature.

\section{Information Sources}

Electronic searches were completed using Agricola (https://search-proquest-com.subzero.lib.uoguelph.ca/ agricola), Medline (OvidSP; http://ovidsp.tx.ovid.com .subzero.lib.uoguelph.ca), and Web of Science (https:// apps.webofknowledge.com.subzero.lib.uoguelph.ca) databases, with the controlled vocabulary option used where available. Grey literature was searched to find unpublished data using Searchable Proceedings of Animal Conferences (S-PAC; https://spac.adsa.org) as well as ProQuest Dissertations and Theses Database (https://search-proquest-com.subzero.lib.uoguelph.ca/ pqdt/dissertations) and Open Access Theses and Dissertations (https://oatd.org/). The literature search was conducted between April 4 and 14, 2017, and limited to English language publications. Search results were uploaded to EndNoteX7 (Clarivate Analytics, Philadelphia, PA) and duplicate results documented and removed. No restriction on publication date was 
placed aside from that of the database (Agricola, 1970; Medline, 1950; Web of Science, 1900; S-PAC, 1935; ProQuest, 1997; Open Access, 1990). Ten relevant studies were preselected by T. F. Duffield and the search results checked to ensure these studies were included. A research librarian (A. Versluis) with the University of Guelph was consulted on the search strategy.

\section{Search Strategy}

Search terms were "calf OR calves OR cattle OR bovine OR dairy OR beef OR Holstein OR Friesian OR Jersey OR ruminant" AND "disbud* OR dehorn* OR cautery OR electric OR rhinehart OR rhinehardt OR iron OR portasol OR express OR buddex OR propane OR butane OR torch" AND "freezing OR numbing OR local OR anesthetic OR anesthetic OR lidocaine OR block OR bupivacaine OR lignocaine OR NSAID OR metacam OR meloxicam OR flunixin OR banamine OR ketoprofen OR anafen OR nonsteroidal anti-inflammatory OR anti-inflammatory OR analgesia OR pain control OR pain mitigation OR meclofenamic acid OR phenylbutasone OR bute OR carprofen OR salicylic acid OR ASA OR aspirin OR naproxen OR tolfenamic acid OR metamizaole sodium."

\section{Study Selection}

Studies were exported from EndNoteX7 into DistillerSR (Evidence Partners Inc., Ottawa, ON, Canada) for 2 rounds of screening. A primary round was conducted independently by C. L. Miltenburg and C. B. Winder, assessing the title and abstract for relevance using the questions:

1) Does the title or abstract describe a primary experimental intervention study?

2) Does the title or abstract describe a study involving calves disbudded by cautery?

3) Does the title or abstract describe one or more of the following intervention groups: local anesthetic, NSAID, or local anesthetic and NSAID?

Studies were excluded if both reviewers agreed that the study did not fulfill 1 or more of these criteria. An unclear option was available for all questions, with the study proceeding to full-text screening if all answers were either yes or unclear. Conflicts between inclusion and exclusion by the 2 reviewers were resolved by consensus. Secondary screening was conducted on the full text of remaining studies by C. L. Miltenburg and C. B. Winder independently, using the initial 3 questions and the following questions:
4) Does the study describe one or more of the following comparator groups: local anesthetic, NSAID, or no pain control?

5) If xylazine (or another sedative) was given, was it given to both intervention and comparator group?

6) Does the study examine at least one of the following outcomes: plasma cortisol concentration, pain behaviors (at least one of ear flick, head shake, head rub, foot stamp, or tail swish), or sensitivity of the horn bud sensitivity as measured by algometer or von Frey monofilaments?

Studies were excluded if both reviewers said no to 1 of the previous questions; conflicts were resolved by consensus. Study citations and reasons for exclusion at this stage of screening were recorded (see Supplemental File S2; https://doi.org/10.3168/jds.2017-14092). Primary screening (questions 1 to 3 ) of title abstracts were pilot tested independently by C. L. Miltenburg and C. B. Winder on the first 100 studies identified by the initial search of Medline (via OvidSP). Full-text screening (questions 4 to 6 ) were pilot tested independently by C. L. Miltenburg and C. B. Winder on 4 studies preselected by T. F. Duffield.

\section{Data Extraction and Data Extraction Items}

Data from studies meeting the study selection criteria were independently extracted by C. L. Miltenburg and C. B. Winder using a standardized form, which was pretested on 4 studies preselected by T. F. Duffield. Discrepancies in data extraction were resolved by consensus.

Study-level data included year published and study period (date or season). Population characteristics consisted of breed, production type (dairy or beef), housing system, commercial or research farm, mean age, sex (male, female, or a mixed group), disbudding method (including disbudding iron type), and disbudding operator (producer, veterinarian, researcher, and so on). Intervention group (including any sham control group) data entailed, for each drug given, drug name, concentration, dose (in $\mathrm{mg}, \mathrm{mL}$, or $\mathrm{mg} / \mathrm{kg}$ ), technique (e.g., cornual nerve block) or route (e.g., i.m., i.v., s.c.), and timing relative to disbudding.

Plasma Cortisol Concentration. Outcomes were extracted as continuous measures with the mean for each treatment group and standard deviation. If this was not available, measures of association were collected with standard error or $95 \%$ confidence interval, and if a statistical model was used all additional variables included were recorded. We collected the number of 
animals in each treatment group, total number of sampling time points, time points relative to disbudding, and whether catheterization or venipuncture was used to obtain samples. If available, data were extracted for each measurement time point individually.

Pain Behaviors. For all individual pain behaviors (ear flick, head shake, head rub, tail swish, foot stamp, and vocalization), outcomes were extracted as continuous measures (number of each behavior within a stated time period) with mean and standard deviation values for each treatment group. If this was not available, measures of association were collected with standard error or $95 \%$ confidence interval, and if a statistical model was used all additional variables were included. If data on pain behaviors were recorded for multiple time periods, the data were extracted for each time period. If pain behaviors were only available as a sum of several behaviors, these data were extracted and the type of behaviors summed was recorded. Data at individual time points were preferred; if only summed data for several time points were available, these were extracted. We collected the number of animals in each treatment group, the total number of observation periods, the time of the observation period relative to disbudding, the length of observation periods, and if observation was done live or by video recording.

Horn Bud Sensitivity. Horn bud sensitivity was defined as a behavioral test where pressure is applied to the area around the horn bud, with a reading taken of the pressure measurement (in kilograms of force) at the time at which the calf reacts by either resisting the restraint or moving away from the device. These data were extracted as a continuous measure with mean and standard deviation values for each treatment group. If this was not available, measures of association were collected with standard error or $95 \%$ confidence interval, and if a statistical model was used all additional variables were included. If data were recorded for multiple time periods, the data were extracted for each time period. We collected the number of animals in each treatment group, the total number of evaluation time points, the type of measurement (algometry or von Frey monofilaments), and measurement time relative to disbudding. Data from individual time points were preferred, but if only summed data from multiple time points were available, these were collected.

\section{Risk of Bias in Individual Studies}

Assessment of bias was done independently by C. L. Miltenburg and C. B. Winder, and was pilot tested by C. L. Miltenburg and C. B. Winder on the same 4 preselected studies chosen by T. F. Duffield for data extraction testing. Disagreements were resolved by con- sensus. Risk of bias was assessed using the Cochrane Collaboration's tool for assessing risk of bias in randomized trials (Higgins et al., 2011), modified by also including an assessment of reporting of randomization (in addition to random sequence generation). Risk of bias was assessed for each outcome class [plasma cortisol concentration, pain behaviors (as a group), and horn bud sensitivity].

\section{Summary Measures and Synthesis of Results}

If more than 2 studies reported the same outcome at a similar time point or period with the same comparison groups, meta-analysis was conducted. Similar time points initially were predefined in the review protocol as not more than 10 min difference during the first 70 min after disbudding and within 20 min after this time. For plasma cortisol concentration and pressure sensitivity, this definition was kept. The original definition of similar time point was also kept for pain behavior measures within the first 60 min after disbudding. However, due to the large variability in time points measuring pain behaviors between studies, time points at $1 \mathrm{~h}$ or later from disbudding were considered similar if measured within $60 \mathrm{~min}$ for the first $3 \mathrm{~h}$ and within 120 min thereafter. Similar time period applied to pain behavior observation windows, the larger of which was initially defined as no more than $150 \%$ of the smaller window. Due to the variability of time periods used in studies measuring pain behaviors, this was expanded to include all time periods, which ranged from 5 to 60 min of observation or video recording per period. If more than 1 outcome measure was reported within a similar time period for a single study, the time closest to the midpoint of the similar time period was used for meta-analysis. This ensured that observations within a similar time period were independent. For outcomes that were measured on the same continuous scale, mean differences were used. If different scales were used (e.g., ear flicks per $10 \mathrm{~min}$ and ear flicks per $15 \mathrm{~min}$ ), standardized mean differences (SMD) were used.

All meta-analysis was done in $\mathrm{R}$ 3.3.3 ( $\mathrm{R}$ Foundation for Statistical Computing, Vienna, Austria) using RStudio version 1.0.136 (RStudio Inc., Boston, MA) with the 'metafor' package (Viechtbauer, 2010). Metaanalysis used a random effects approach, and weighting of primary studies was done using the inverse variance method. Random effects models were chosen, as it was assumed study-specific differences may exist and thus studies may not all measure the same true effect. Heterogeneity between studies was assessed with the $I^{2}$ statistic (Viechtbauer, 2010). Heterogeneity was to be explored via subgroup analysis or meta-regression if enough studies were found for a single outcome. 
A subgroup analysis was planned for those with and without the use of xylazine sedation for each intervention comparator group (if there were at least 3 studies in each group). For treatment group comparisons of outcomes lacking at least 2 studies, data were reported as a narrative synthesis (Table 1 ).

\section{Risk of Bias at the Review Level}

If 10 or more studies were found for a single outcome, a funnel plot (effect estimate versus the inverse of its standard error) was used to visually assess potential for publication bias (Viechtbauer, 2010).

\section{RESULTS}

\section{Study Selection and Characteristics}

Results of the search strategy and study selection are presented in Figure 1. Seventy-five full-text articles were reviewed, with 54 not meeting eligibility criteria and 21 articles containing 23 separate experiments included in the qualitative synthesis. Details of the 21 studies remaining after full-text screening are listed in Table 1. A list of studies excluded at the full-text screening stage is available in Supplemental File S3 (https://doi .org/10.3168/jds.2017-14092).

\section{Relevant Interventions and Comparator Groups}

Of the 21 included studies, 12 contained an intervention group receiving local anesthetic with a comparator of saline or no treatment; these comprised 13 local anesthetic treatment groups. Fourteen studies included an intervention group receiving an NSAID in addition to local anesthetic with a comparator of local anesthetic only; these compromised 22 treatment groups. One study had a treatment group of calves receiving NSAID alone with a comparator group of no treatment (Stilwell, 2009). Two studies used xylazine sedation given to both intervention and comparator groups; one of these studies compared local anesthetic to saline (Stilwell et al., 2010), whereas the other compared local anesthetic and ketoprofen to local anesthetic alone (Faulkner and Weary, 2000).

\section{Data Extraction from Figures}

Data extraction deviated from the a priori protocol for those studies not reporting values numerically, but which had data available as a graph with a measure of variation. These values were extracted independently by both C. L. Miltenburg and C. B. Winder using WebPlotDigitizer version 3.12 (Rohatgi, 2017). Initial differences between values were discussed and graphs were re-examined to ensure errors had not been made; further, smaller, discrepancies in values were averaged. Plasma cortisol data from 10 of 15 studies were only available graphically, as were data from 5 of 7 studies reporting pain behaviors and data from all 4 studies reporting pressure sensitivity.

\section{Synthesis of Study Results by Outcome Type}

Forest plots for all meta-analyses conducted are available in Supplemental File S4 (https://doi.org/10 .3168/jds.2017-14092); a summary of the overall effect measures for each meta-analyses conducted for different time points for the same intervention/comparator and the same outcome are included as figures (Figure $2,3,4,5$, and 6 ).

Plasma Cortisol Concentration. Although 18 studies reported measuring plasma cortisol concentration, only 15 were included in meta-analyses. GrøndahlNielsen et al. (1999) reported only maximum cortisol values. Stock et al. (2015) reported maximum cortisol values, area under the effect curve for 0 to 24,24 to 96 , and 0 to $96 \mathrm{~h}$ postdisbudding, and percent change in cortisol from baseline over the same time periods. Huber et al. (2013) did not report any measure of variability. Therefore, these 3 studies were excluded from the meta-analysis.

Sampling was done via jugular venipuncture in 7 studies, jugular catheters in 7 studies, and was unreported in 1 study. Units of measurement were not uniform between studies; absolute values (in both nmol/L and ng/ $\mathrm{mL}$ ) were reported as well as change from baseline and back-transformed geometric means. As a result, SMD were used for all plasma cortisol-related meta-analyses.

For studies included in the meta-analyses, time points were considered as described above, where 3 or more studies compared the same intervention at a similar time. For the comparison of local anesthetic to saline or no treatment, 7 treatment groups were used at 30 min, 8 treatment groups at $1 \mathrm{~h}, 4$ treatment groups at $2 \mathrm{~h}, 5$ treatment groups at $3 \mathrm{~h}, 5$ treatment groups at $4 \mathrm{~h}, 4$ treatment groups at $6 \mathrm{~h}$, and 4 treatment groups at $24 \mathrm{~h}$ postdisbudding. For studies reporting plasma cortisol concentration at $30 \mathrm{~min}$ or $1 \mathrm{~h}$ postdisbudding, a significant protective effect of local anesthetic was seen (Figure 2), but substantial heterogeneity existed among studies $\left(I^{2}>50 \%\right)$. For studies reporting plasma cortisol concentration at 2 or $3 \mathrm{~h}$ postdisbudding, no effect of treatment on cortisol was observed and heterogeneity was moderate $\left(I^{2}=43 \%\right.$ for both 2 and $\left.3 \mathrm{~h}\right)$. At $4 \mathrm{~h}$ postdisbudding, treatment with local anesthetic resulted in higher cortisol concentrations than saline or no treatment and substantial heterogeneity among 
WINDER ET AL.

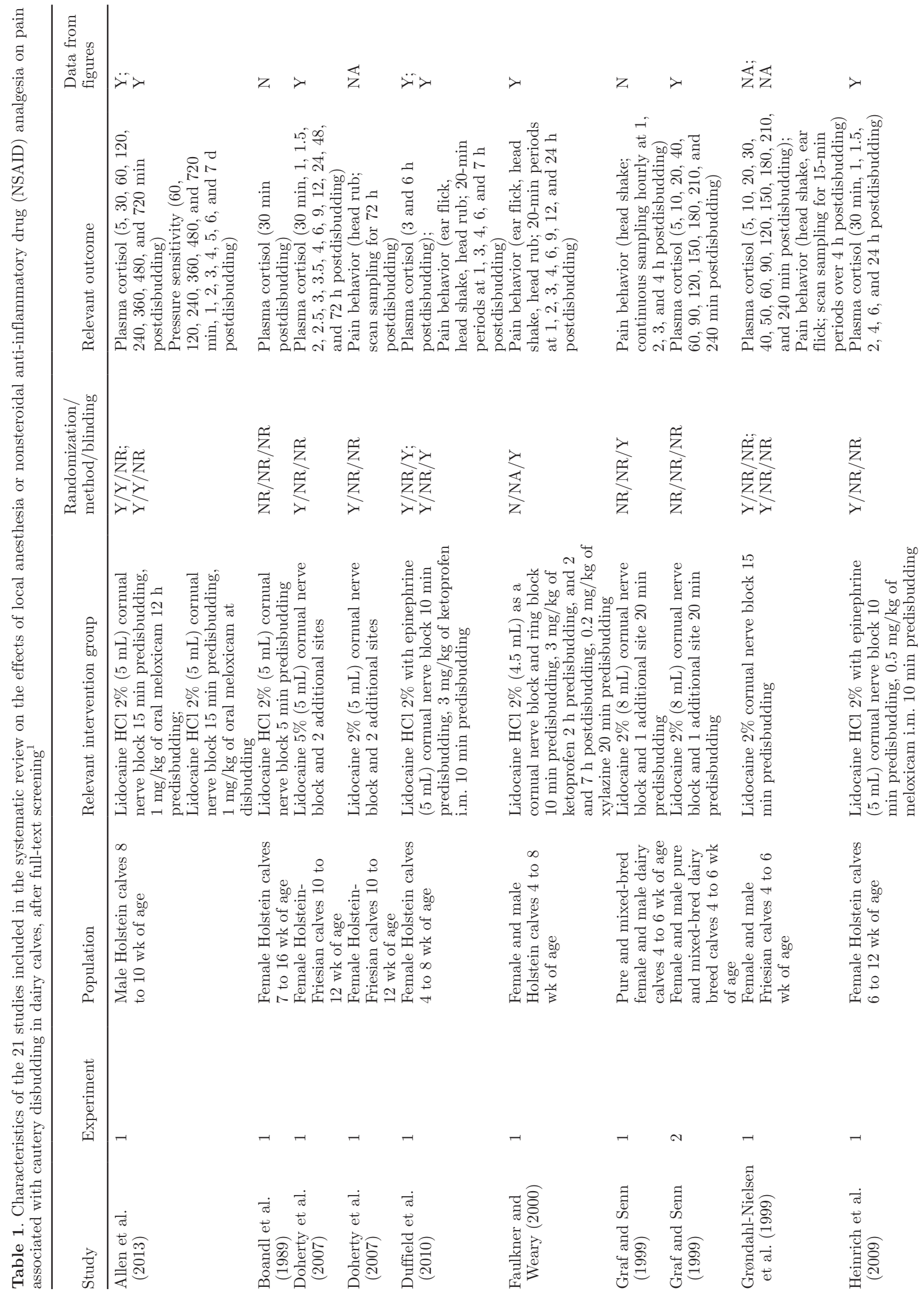


PAIN CONTROL FOR DISBUDDING: A SYSTEMATIC REVIEW

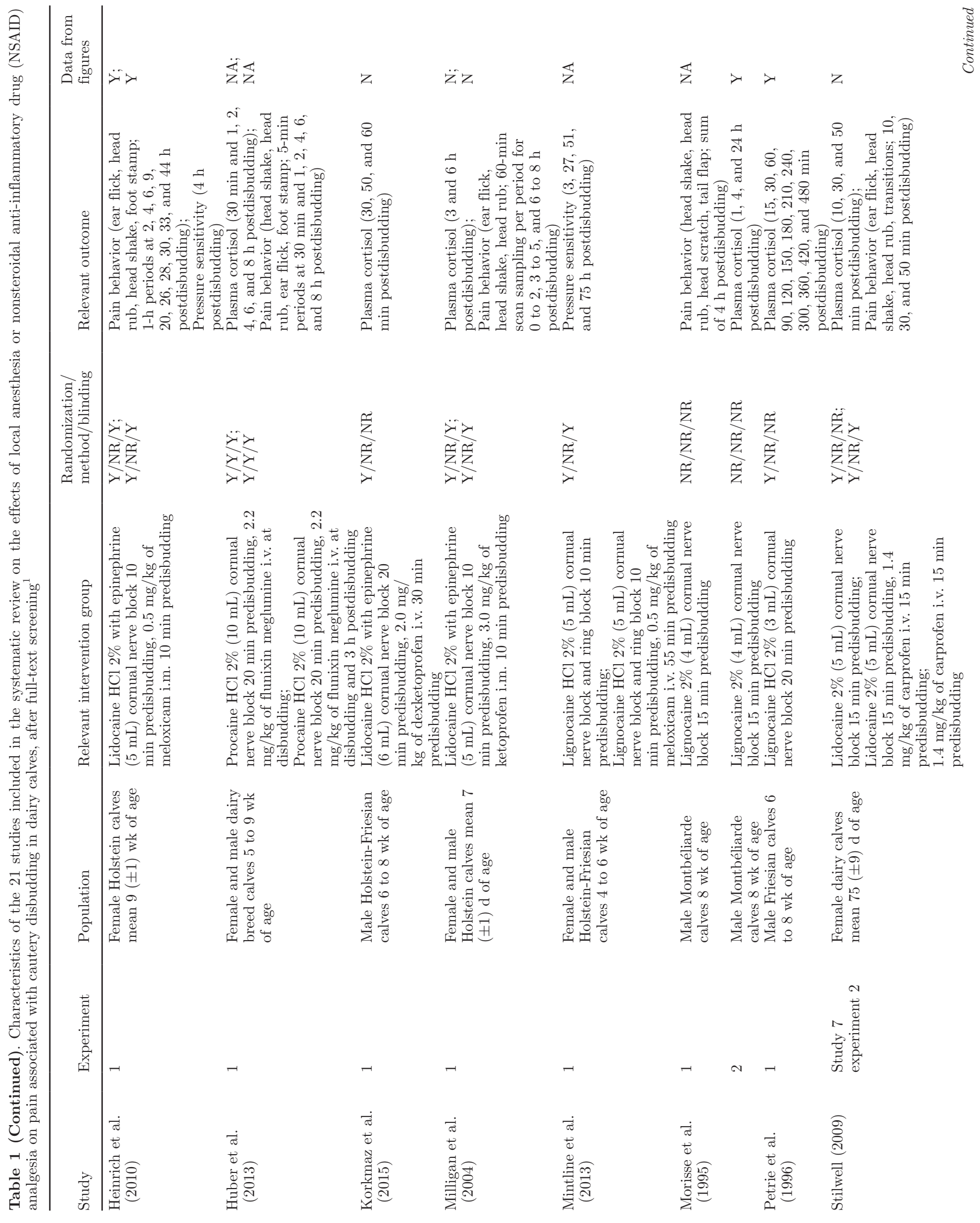


WINDER ET AL.

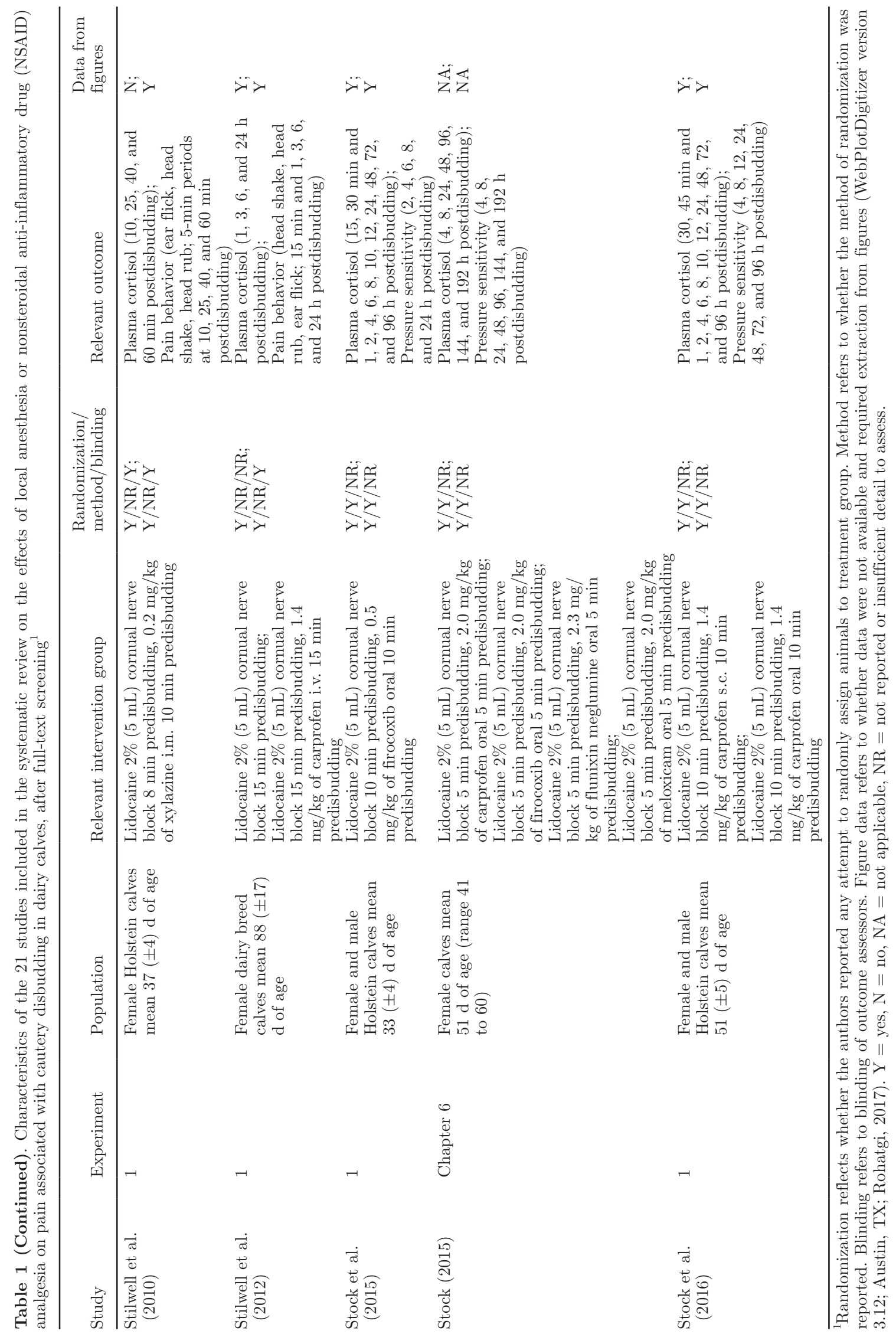


studies was present $\left(I^{2}>50 \%\right)$. For studies reporting cortisol at $6 \mathrm{~h}$ or $24 \mathrm{~h}$, we found no effect of treatment and heterogeneity was again present $\left(I^{2}>50 \%\right)$.

For the comparison of local anesthetic and NSAID to local anesthetic alone, 8 treatment groups were used at
30 min, 9 treatment groups were used at 1 h, 6 treatment groups were used at $2 \mathrm{~h}, 3$ treatment groups at 3 h, 6 treatment groups at $4 \mathrm{~h}$, and 9 treatment groups at $6 \mathrm{~h}$, and 7 treatment groups at $24 \mathrm{~h}$ postdisbudding. For studies reporting plasma cortisol at $30 \mathrm{~min}$ and 1 ,

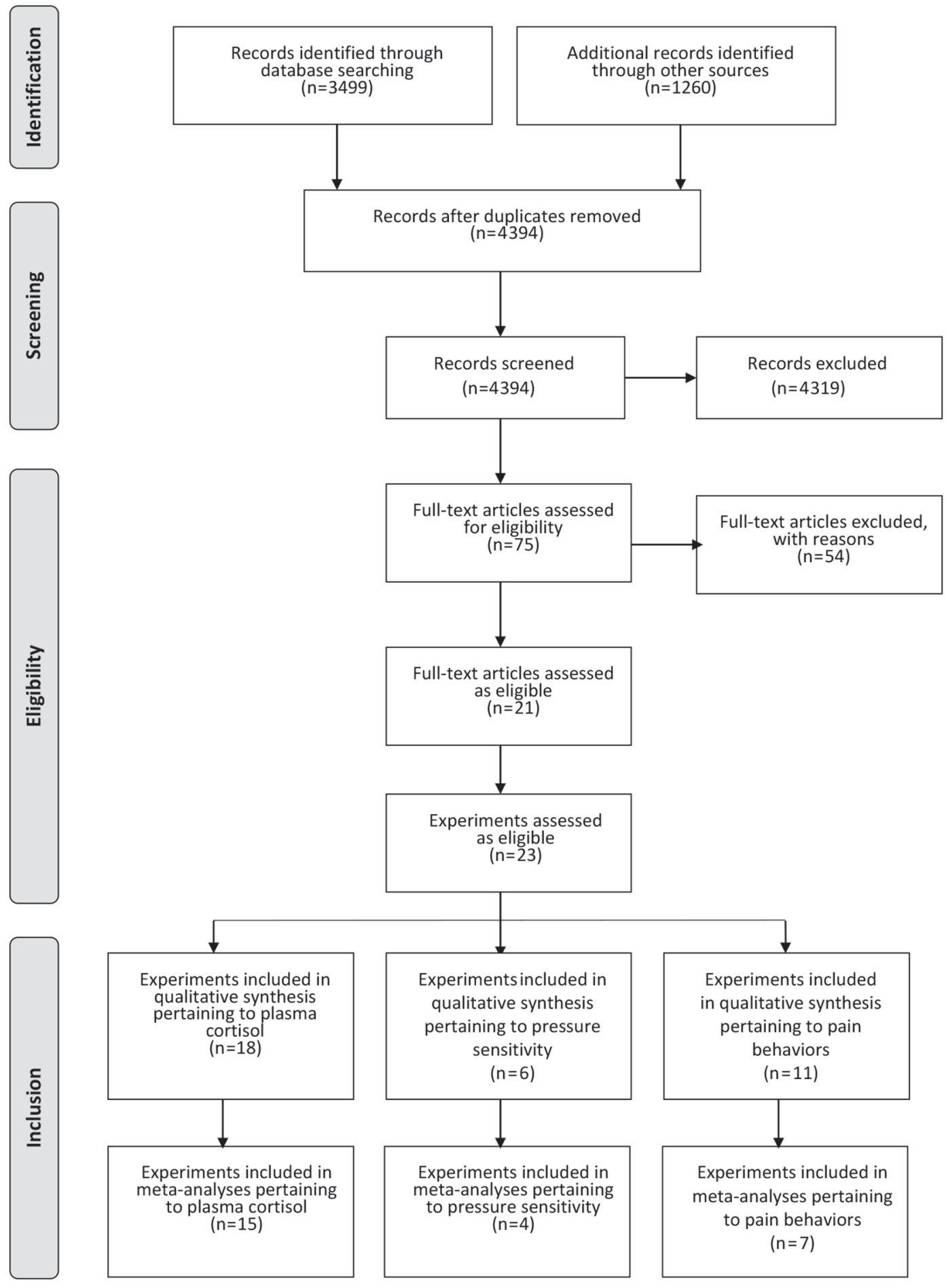

Figure 1. Preferred Reporting Items for Systematic Reviews and Meta-Analyses (PRISMA) study flow diagram (Moher et al., 2010). 
2 , or $3 \mathrm{~h}$ postdisbudding, no effect of treatment and no heterogeneity was observed $\left(I^{2}=0 \%\right.$; Figure 3$)$. For studies reporting cortisol at $4 \mathrm{~h}$ postdisbudding, a protective effect was detected, with moderate heterogeneity between studies $\left(I^{2}=44 \%\right)$. For studies reporting at $6 \mathrm{~h}$ postdisbudding, no overall treatment effect was present and heterogeneity was low $\left(I^{2}=17 \%\right)$. At 24 h postdisbudding, plasma cortisol concentration was greater in calves that received local anesthesia and NSAID and we noted moderate heterogeneity $\left(I^{2}=46 \%\right)$.

Pressure Sensitivity. Six studies reported measuring pressure sensitivity, either using a pressure algometer (Heinrich et al., 2010; Allen et al., 2013; Stock, 2015; Stock et al., 2015, 2016) or VonFrey monofilaments (Mintline et al., 2013). Two of these studies were not used in the meta-analyses. Mintline et al. (2013) did not report a measure of variability among treatment groups. Stock (2015) reported percent change in algometry score at 24 and $192 \mathrm{~h}$ and could not be combined. Three studies reported absolute values (kgf) whereas 1 reported back-transformed geometric means, and therefore SMD were used in pressure sensitivity related meta-analyses.

Of the 4 studies included in the meta-analyses, only comparisons between local anesthesia with NSAID and local anesthesia alone were examined. Time points were considered as described in the methods, where 3 or more studies compared the same intervention at a similar time. Three treatment groups were used at $2 \mathrm{~h}$, 6 treatment groups at $4 \mathrm{~h}, 3$ treatment groups at $6 \mathrm{~h}$, 5 treatment groups at $8 \mathrm{~h}, 4$ treatment groups at $12 \mathrm{~h}$, 5 treatment groups at $24 \mathrm{~h}$, and 4 treatment groups at 48,72 , and $96 \mathrm{~h}$ postdisbudding (Figure 4). For studies reporting pressure sensitivity at $2 \mathrm{~h}$ postdisbudding, no effect of treatment was seen and moderate heterogeneity was present between studies $\left(I^{2}=37 \%\right)$. For studies reporting at 4 or $6 \mathrm{~h}$ postdisbudding, an overall effect was seen with calves treated with NSAID in addition to local anesthetic tolerating more pressure on the areas around their horn bud. No heterogeneity was seen

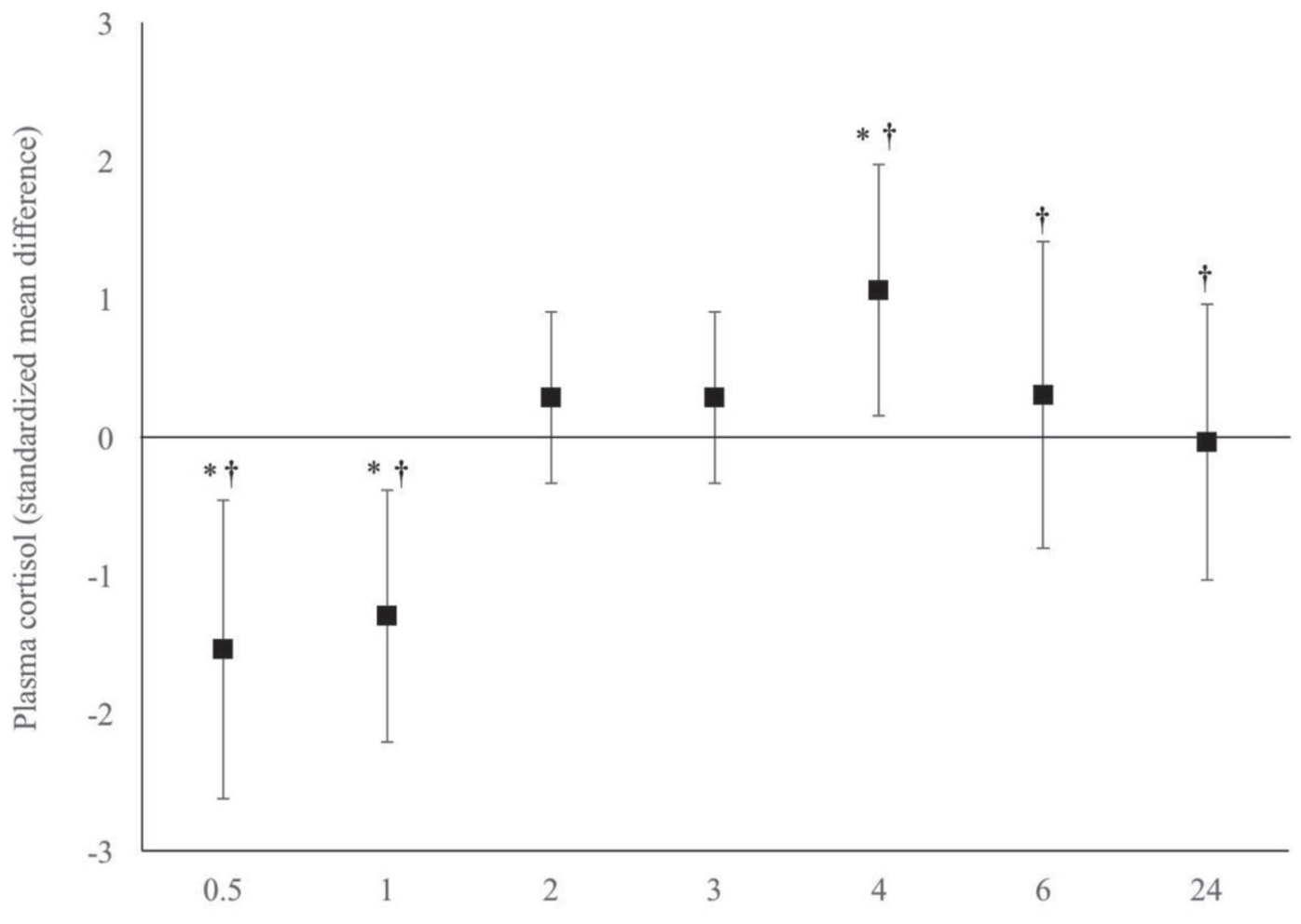

Hours post-disbudding

Figure 2. Overall effect measures $( \pm 95 \% \mathrm{CI})$ of random effects meta-analyses of the effect of local anesthesia compared with control on standardized mean difference in plasma cortisol at time points $0.5 \mathrm{~h}$ ( 5 studies; 7 comparison groups; 122 calves; $\left.I^{2}=83 \%\right), 1 \mathrm{~h}(7$ studies; 8 comparison groups; 135 calves; $I^{2}=80 \%$ ), 2 h ( 4 studies; 5 comparison groups; 76 calves), $3 \mathrm{~h}$ ( 4 studies; 5 comparison groups; 76 calves), 4 h (4 studies; 5 comparison groups; 87 calves; $\left.I^{2}=72 \%\right), 6 \mathrm{~h}$ (3 studies; 4 comparison groups; 60 calves; $\left.I^{2}=75 \%\right)$, and $24 \mathrm{~h}(3$ studies, 4 comparison groups; 69 calves; $\left.I^{2}=74 \%\right)$ postdisbudding. An asterisk $(*)$ indicates a significant overall effect of treatment $(P<0.05)$; a dagger $(\dagger)$ indicates substantial heterogeneity $\left(I^{2}>50 \%\right)$ within a meta-analysis. 
between studies at these 2 time points $\left(I^{2}=0 \%\right)$. For all further time points $(8,12,24,48,72$, or $96 \mathrm{~h})$, no effect of treatment was observed, and we noted low heterogeneity between studies $\left(I^{2}=0-20 \%\right)$.

Pain Behavior. Eleven studies reported measuring at least 1 pain-related behavior (ear flick, head shake, head rub, tail swish, foot stamp, or vocalization); 7 of these studies were included in the meta-analyses. Stilwell (2009) reported combined values for ear flick, head shake, head rub, and quick transitions from standing to laying and could not be combined with results from other studies. Morisse et al. (1995) reported total frequency of behaviors by treatment group for the first $4 \mathrm{~h}$ postdisbudding, with no measure of variability. Huber et al. (2013) and Grøndahl-Nielsen et al. (1999) also did not report any measures of variability.

Three studies with 3 treatments (Graf and Senn, 1999; Stilwell et al., 2010; Stilwell et al., 2012) compared local anesthesia and saline or no treatment. Both studies by Stilwell et al. (2010; 2012) reported ear flick, head shake, and head rub using live observation over
5- (Stilwell et al., 2010) and 15-min (Stilwell et al., 2012) periods. Graf and Senn (1999) reported head shakes using video recording over 60 -min periods. Only 1 time point for 1 pain behavior, head shakes at $1 \mathrm{~h}$ postdisbudding, could be combined in a meta-analysis. This synthesis showed no overall treatment effect of reduction in mean head shakes $(\mathrm{SMD}=-0.58 ; 95 \% \mathrm{CI}$ $=-1.17-0.01)$, with low heterogeneity between studies $\left(I^{2}=11 \%\right)$.

Five studies with 5 treatments compared local anesthesia and NSAID to local anesthesia alone; 3 used video recording whereas 2 employed live observations. Recording period length ranged from 15 to $60 \mathrm{~min}$. Three studies reported ear flick, head shake, and head rub, 1 reported ear flick and head shake, and 1 reported only ear flick. For ear flick, 4 treatment groups were compared at $1 \mathrm{~h}, 3$ treatment groups at $3 \mathrm{~h}, 4$ treatment groups at $4 \mathrm{~h}, 5$ treatment groups at $6 \mathrm{~h}$, and 3 treatment groups at $24 \mathrm{~h}$ postdisbudding (Figure 5). For studies reporting ear flicks at $1 \mathrm{~h}$ postdisbudding, no treatment effect was seen, with substantial hetero-

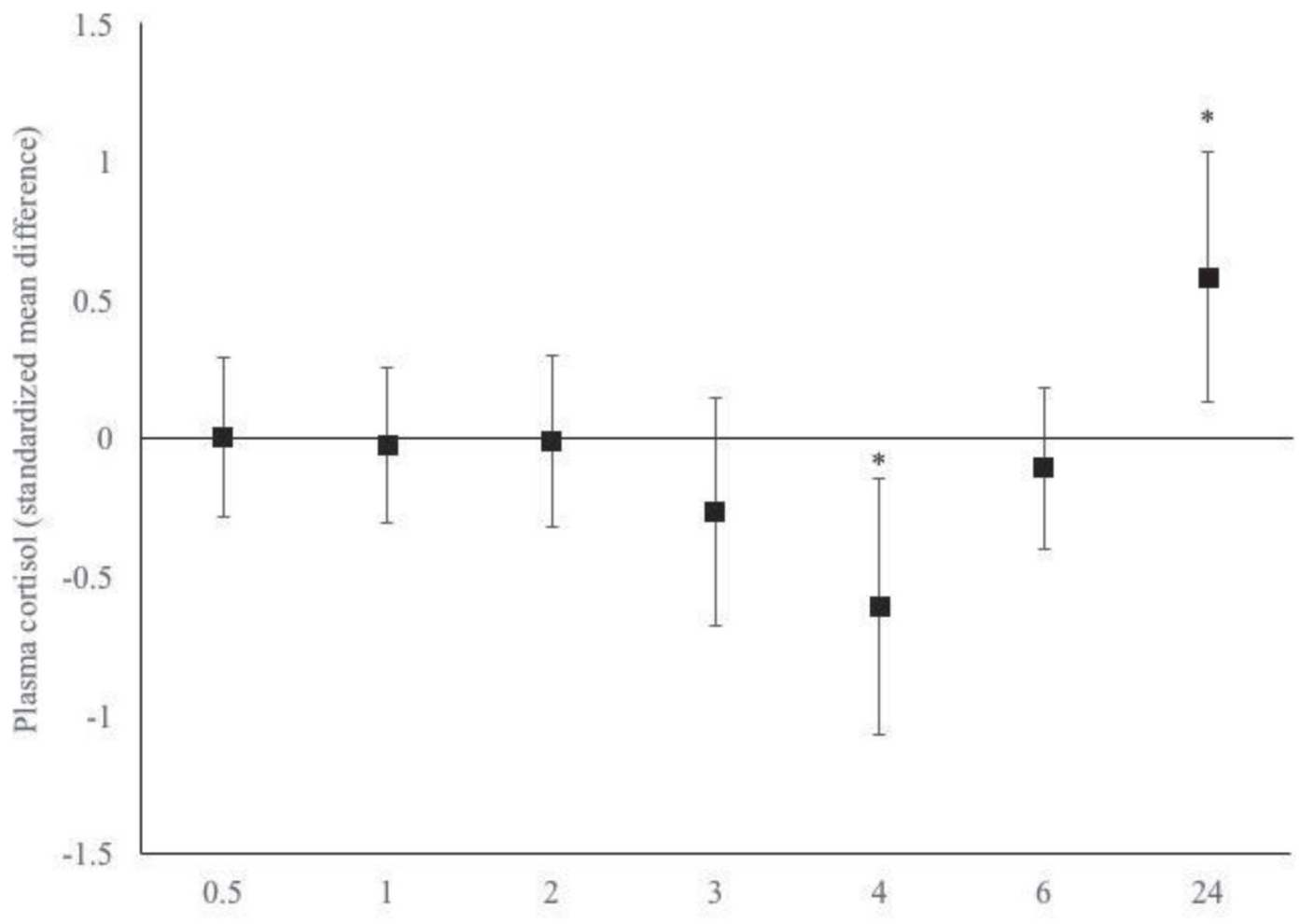

Hours post-disbudding

Figure 3. Overall effect measures $( \pm 95 \% \mathrm{CI})$ of random effects meta-analyses of the effect of local anesthesia and nonsteroidal anti-inflammatory drugs (NSAID) compared with local anesthesia on standardized mean difference in plasma cortisol at time points 0.5 h (6 studies; 8 comparison groups; 186 calves), 1 h ( 7 studies; 9 comparison groups; 198 calves), 2 h ( 4 studies; 6 comparison groups; 160 calves), 3 h (3 studies; 3 comparison groups; 92 calves), $4 \mathrm{~h}$ (4 studies; 6 comparison groups; 160 calves), $6 \mathrm{~h}$ ( 7 studies; 9 comparison groups; 252 calves), and $24 \mathrm{~h}$ ( 5 studies, 7 comparison groups; 172 calves; $\left.I^{2}=46 \%\right)$ postdisbudding. An asterisk $\left(^{*}\right)$ indicates a significant overall effect of treatment $(P<0.05)$. 
geneity $\left(I^{2}=90 \%\right)$. For studies reporting ear flicks at 3 or $4 \mathrm{~h}$, a protective effect of treatment was seen, with low heterogeneity $\left(I^{2}=22\right.$ and $0 \%$ for 3 and $4 \mathrm{~h}$, respectively). At 6 or $24 \mathrm{~h}$, no overall effect of treatment was measured and substantial heterogeneity was present between studies $\left(I^{2}>50 \%\right)$.

For head shake, 3 treatment groups were compared at $1 \mathrm{~h}, 3$ treatment groups at $4 \mathrm{~h}, 4$ treatment groups at 6 $\mathrm{h}$, and 3 at $24 \mathrm{~h}$ postdisbudding (Figure 6 ). For studies reporting head shake at $1 \mathrm{~h}$ postdisbudding, no effect of treatment was seen, with no heterogeneity present between studies $\left(I^{2}=0 \%\right)$. For studies reporting at 4 or $6 \mathrm{~h}$ postdisbudding, a protective effect of treatment was found with no heterogeneity between studies $\left(I^{2}=\right.$ $0 \%$ ). For studies reporting at $24 \mathrm{~h}$ postdisbudding, no overall treatment effect was seen, although substantial heterogeneity was present between studies $\left(I^{2}=59 \%\right)$.

For head rub, 3 treatment groups were compared at $6 \mathrm{~h}$ postdisbudding and no effect of treatment was observed $(\mathrm{SMD}=-0.24 ; 95 \% \mathrm{CI}=-0.71-0.23)$. No heterogeneity was detected between studies $\left(I^{2}=0 \%\right)$.

\section{Subgroup Analysis, Meta-Regression, and Publication Bias}

Xylazine sedation was identified a priori as a possible subgroup of studies; however, as only 2 studies were included with this treatment, each with a different intervention or comparator group, no subgroup analysis was possible. During data extraction the NSAID treatments were recorded for possible subgroup analysis. Too much variability was present to have enough similar treatments for subgroup analysis, however (see Table 2). Six NSAID products were given via 4 routes, in some cases at different dosages, and at a range of time points. As fewer than 10 studies were used in all meta-analyses, no meta-regression was attempted, nor were funnel plots used to detect possible publication bias.

\section{Assessment of Risk of Bias Across Studies}

All studies failed to report pertinent information in at least 1 section in the risk of bias assessment. Al-

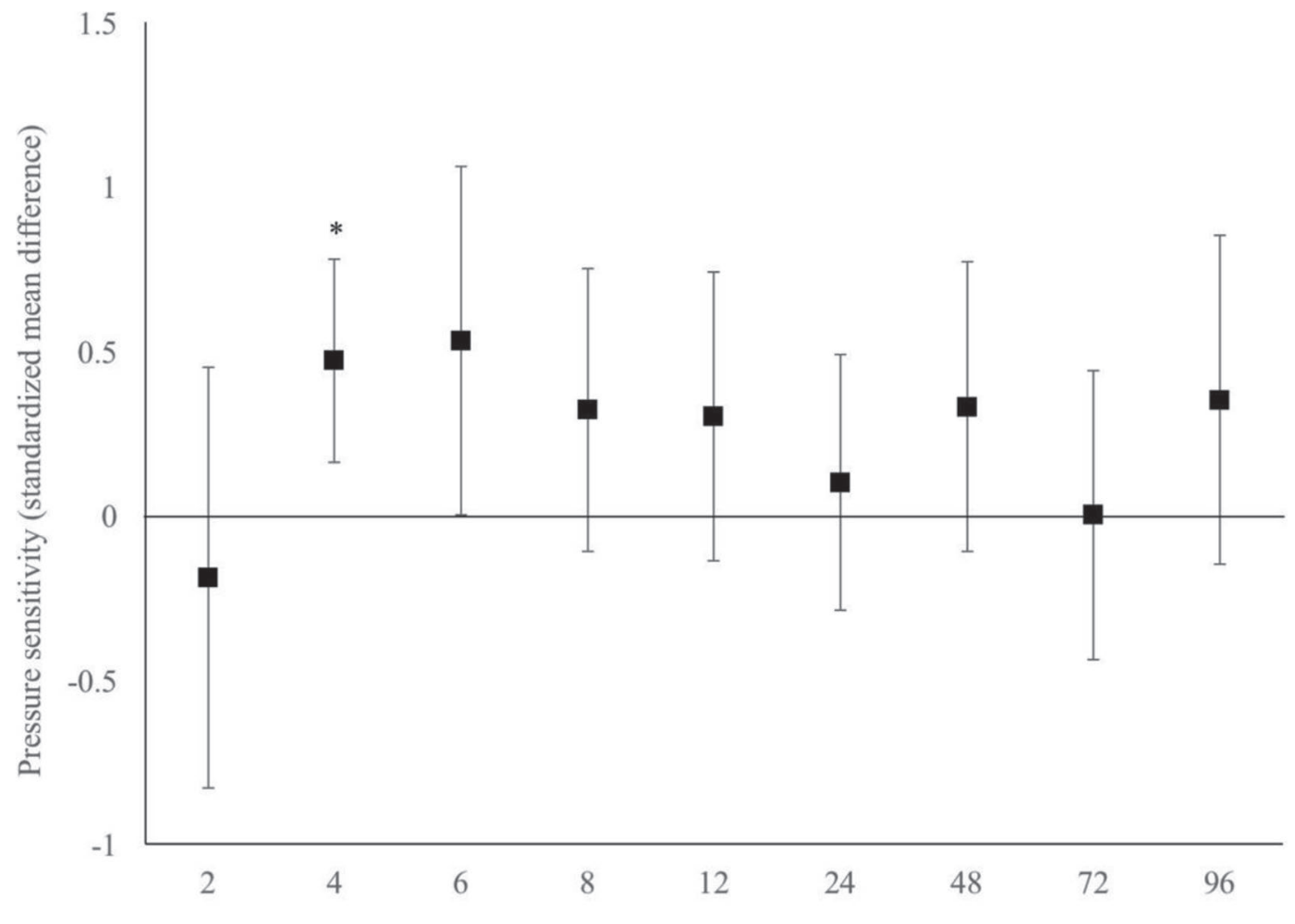

Hours post-disbudding

Figure 4. Overall effect measures $( \pm 95 \% \mathrm{CI})$ of random effects meta-analyses of the effect of local anesthesia and nonsteroidal anti-inflammatory drugs (NSAID) compared with local anesthesia on standardized mean difference in horn bud pressure sensitivity at time points 2 h (2 studies; 3 comparison groups; 60 calves), 4 h (4 studies; 6 comparison groups; 160 calves), 6 h (2 studies; 3 comparison groups; 60 calves), 8 h ( 3 studies; 5 comparison groups; 100 calves), 12 h (2 studies; 4 comparison groups; 80 calves), 24 h (3 studies; 5 comparison groups; 100 calves), 48 h ( 2 studies; 4 comparison groups; 80 calves), 72 h ( 2 studies; 4 comparison groups; 80 calves), and 96 h ( 2 studies; 4 comparison groups; 80 calves) postdisbudding. An asterisk $(*)$ indicates a significant overall effect of treatment $(P<0.05)$. 


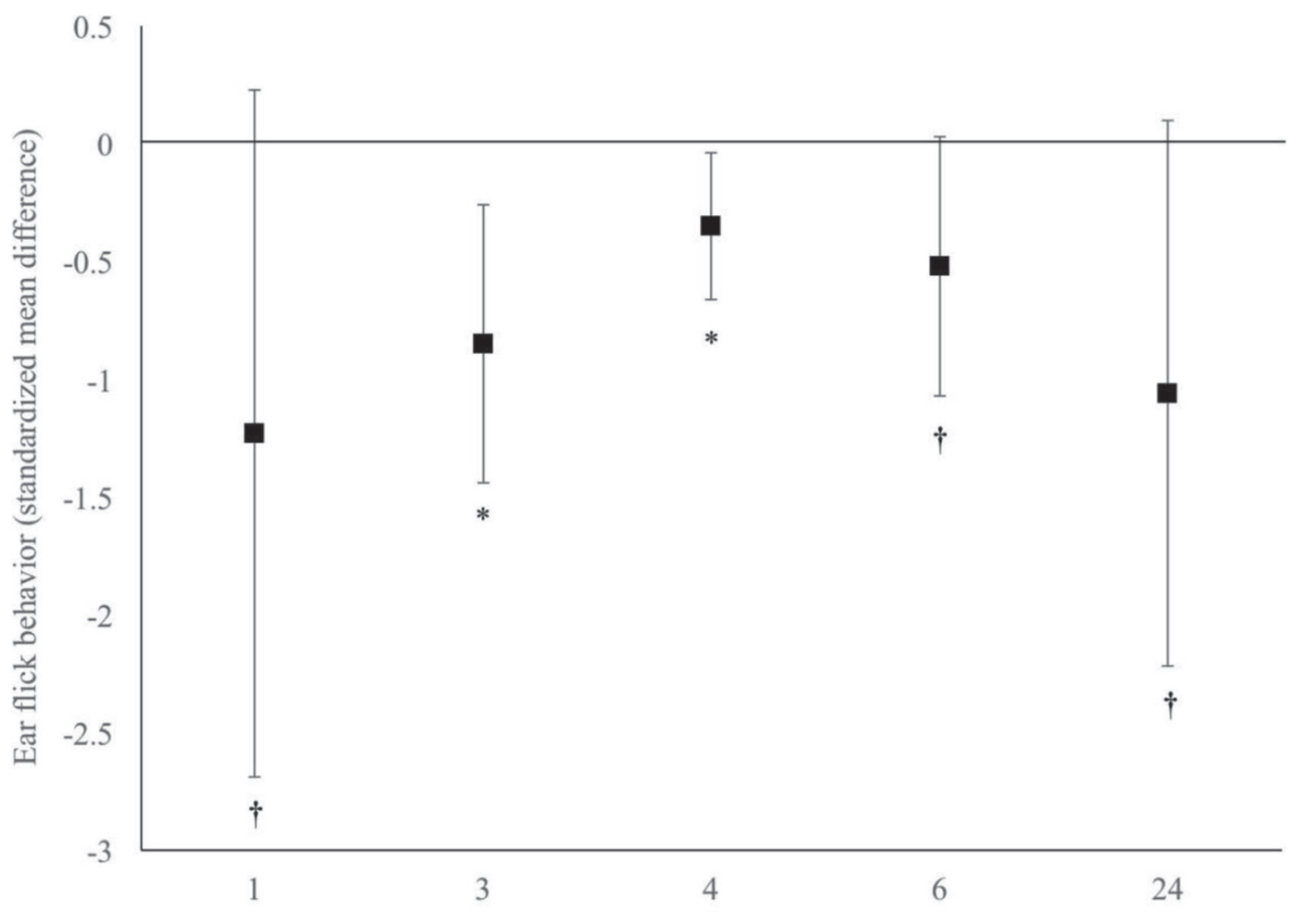

Hours post-disbudding

Figure 5. Overall effect measures $( \pm 95 \% \mathrm{CI})$ of random effects meta-analyses of the effect of local anesthesia and nonsteroidal anti-inflammatory drugs (NSAID) compared with local anesthesia on standardized mean difference in ear flick behavior at time points $1 \mathrm{~h}$ ( 4 studies; 4 comparison groups; 132 calves; $I^{2}=90 \%$ ), 3 h (3 studies; 3 comparison groups; 72 calves), $4 \mathrm{~h}$ ( 4 studies; 4 comparison groups; 160 calves), 6 h ( 5 studies; 5 comparison groups; 172 calves; $\left.I^{2}=63 \%\right)$, and $24 \mathrm{~h}$ ( 3 studies; 3 comparison groups; 92 calves; $\left.I^{2}=78 \%\right)$ postdisbudding. An asterisk $(*)$ indicates a significant overall effect of treatment $(P<0.05)$; a dagger $(\dagger)$ indicates substantial heterogeneity $\left(I^{2}>50 \%\right)$ within a meta-analysis.

though 17 studies reported randomization of calves to treatment groups, 12 did not report the method of randomization. Method of allocation concealment was only reported in 1 study. Blinding of outcome assessment was reported for 19 of 35 outcomes. Whereas data may or may not have been combinable based on how they were reported, all studies did report some form of outcome data for all outcomes listed in the studies' materials and methods sections.

\section{DISCUSSION}

\section{Effect of Local Anesthetic Compared with Saline or No Treatment}

Our findings illustrate that the provision of local anesthetic was associated with an initial protective effect on plasma cortisol, with a negative effect seen in studies evaluating cortisol after the duration of anesthesia, after which treatment and control groups were not different. This rise in cortisol observed in the treatment group after anesthesia has been postulated to be due to inflammatory pain, which may be reduced in control calves, as their initial cortisol spike may result in a dampening of the inflammatory response as compared with calves without this initial rise (Stock et al., 2013).

The heterogeneity among studies at $30 \mathrm{~min}$ and 1, 4, 6 , and $24 \mathrm{~h}$ postdisbudding, indicated inconsistency of effect between studies, which may be due to numerous factors, including random variation as well as contextual and methodological variation. Even with 7 and 8 studies included in the meta-analyses, total sample size was only 60 to 70 calves per treatment group; random variation within these populations due to the small sample size may account for some of the between-study variability. Additionally, plasma cortisol could have been influenced by many other factors than use of pain control, such as differences in handling methods, time of day of sample collection, diameter of disbudding iron, and resultant wound size, as well as factors associated with the pain control itself (percentage of active ingredient, volume used, technique used, if epinephrine 


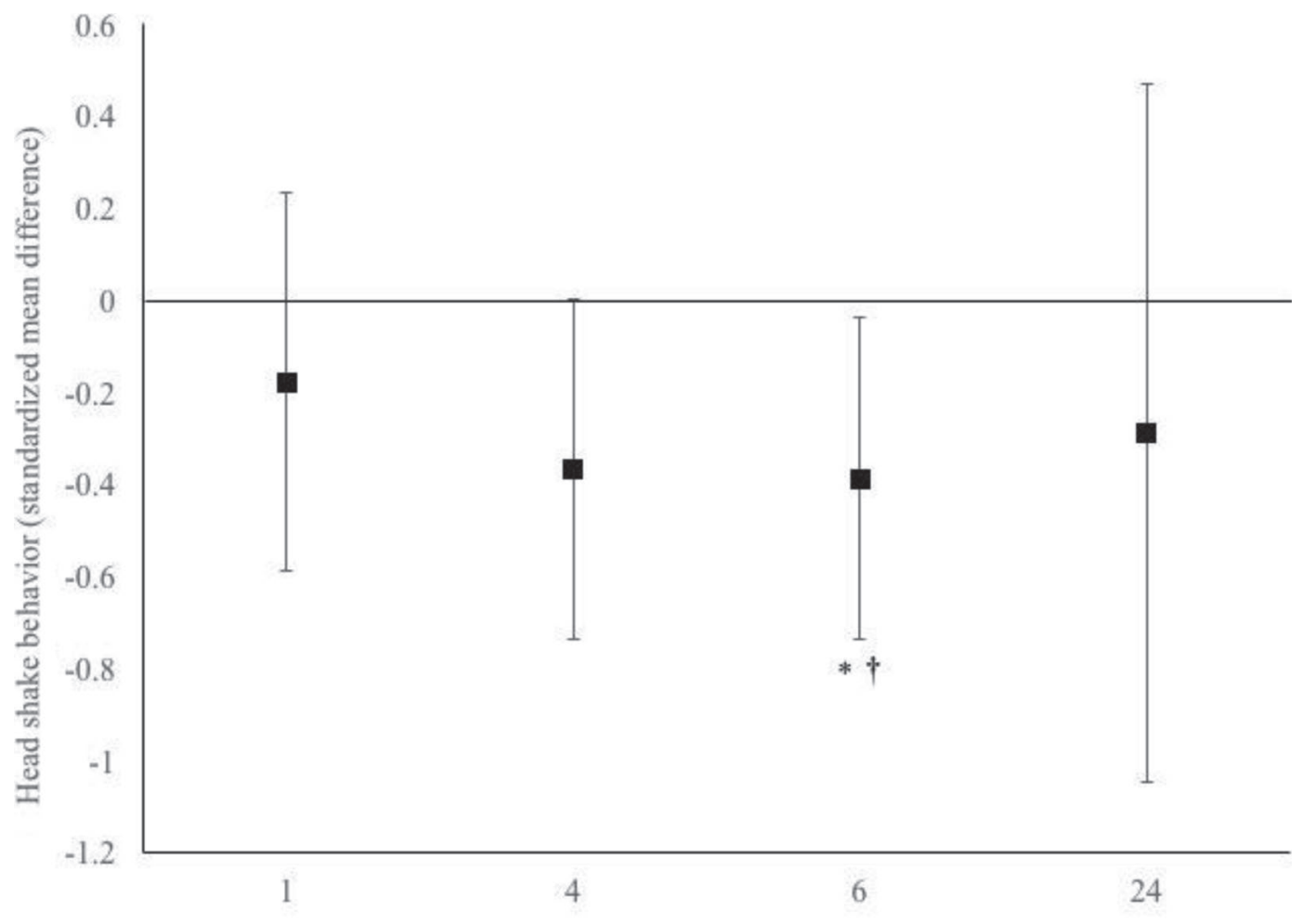

Hours post-disbudding

Figure 6. Overall effect measures $( \pm 95 \% \mathrm{CI})$ of random effects meta-analyses of the effect of local anesthesia and nonsteroidal anti-inflammatory drugs (NSAID) compared with local anesthesia on standardized mean difference in head shake behavior at time points $1 \mathrm{~h}$ (3 studies; 3 comparison groups; 92 calves), $4 \mathrm{~h}$ (3 studies; 3 comparison groups; 120 calves), $6 \mathrm{~h}$ (4 studies; 4 comparison groups; 132 calves), and 24 h (3 studies; 3 comparison groups; 92 calves) post-disbudding. An asterisk $(*)$ indicates a significant overall effect of treatment $(P<0.05)$; a dagger $(\dagger)$ indicates substantial heterogeneity (percentage of variation across studies beyond chance, $\mathrm{I}^{2}>50 \%$ ) within a meta-analysis.

Table 2. Nonsteroidal anti-inflammatory drug (NSAID) protocols among the 17 treatment trials that compared local anesthetic and NSAID to local anesthetic alone, which were included after full-text screening

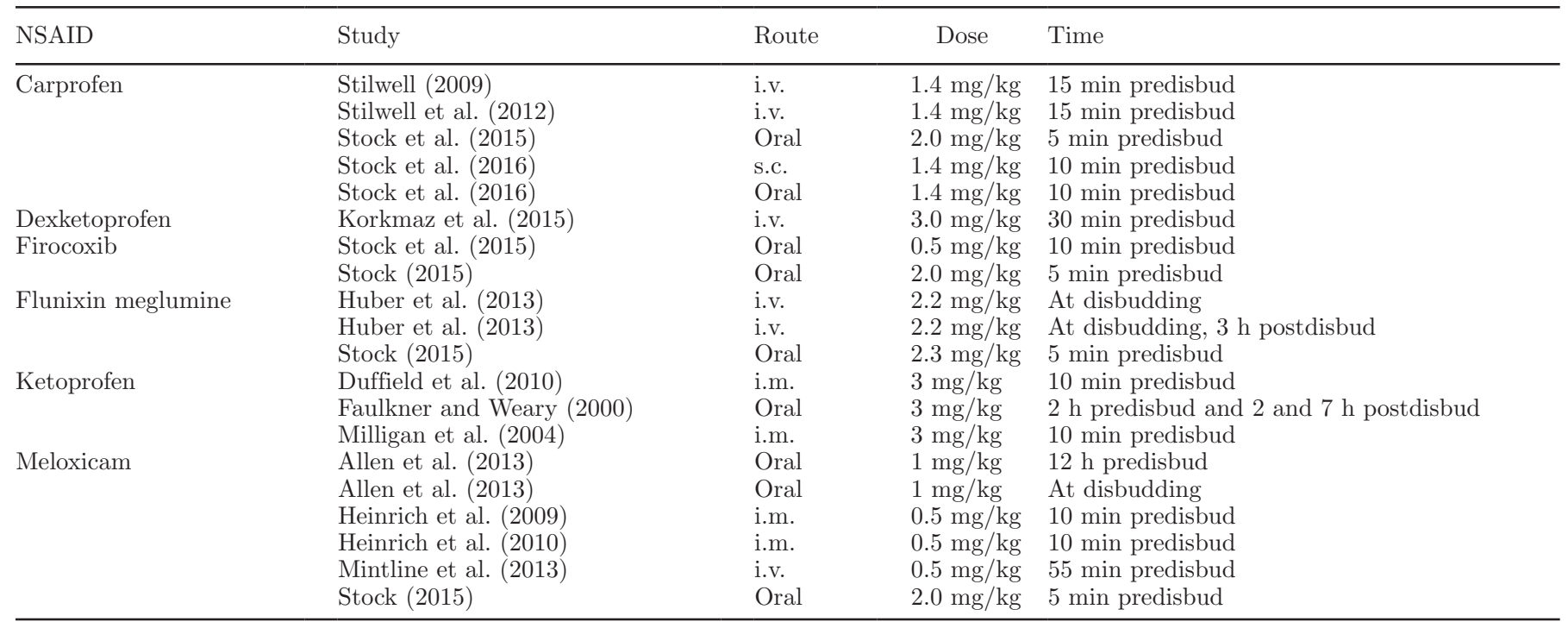


was included, and so on). Whereas these would likely be nondifferential within a study, it is possible some of these factors may interact with the effect of treatment, resulting in variability between effect magnitude or duration between studies. Depending on the nature of the disbudding insult, the delayed rise in cortisol may last a different length of time, which may account for some discrepancies in the 4 and $6 \mathrm{~h}$ meta-analyses. To fully explore any of these possible causes of heterogeneity, a larger number of studies would be required. Additionally, although care was taken to avoid errors in extracting data from graphs, this may have added to the observed heterogeneity between studies based on the resolution of the images available.

\section{Effect of Local Anesthetic and NSAID Compared with Local Anesthetic Alone}

Overall, beneficial effects were found at 3,4 , and $6 \mathrm{~h}$ postdisbudding when NSAID was given in addition to local anesthetic. This likely corresponds to the time after the duration of effect of the local anesthetic, and may be due to a reduction in inflammatory pain. Whereas a beneficial effect was seen at various time points for all classes of outcome (plasma cortisol concentration, pain behavior, and pressure sensitivity), these effects did not necessarily occur at the same time points for each outcome, and heterogeneity was substantial in some analyses, including several using pain behavior outcomes. The primary studies included in these meta-analyses vary between analyses (not all studies reported at all included time points), and therefore differences in time to effect by outcome may be a function of the outcome itself as well as study design, NSAID used, volume and route given, local anesthetic used, volume and route given, and specific characteristics of the different study populations. Similar to the findings in the comparison of local anesthetic to no treatment, sample sizes in many individual studies were quite small (ranging from 6 to 30 calves per group), which increased the chance for random variation in effect found between studies, although confidence intervals will also be wide. A lack of heterogeneity in many of the analyses pertaining to pressure sensitivity may reflect less inherent variability in this outcome compared with others, or it may be in part due to the similarity in study design and treatment, as most trials in these analyses were done by the same research group.

At $24 \mathrm{~h}$, a protective effect of no treatment on plasma cortisol occurred, with moderate heterogeneity between studies. Other outcome measures at $24 \mathrm{~h}$ did not find this, and individual studies reporting at later time points did not see a continuation of this trend (Allen et al., 2013; Stock, 2015; Stock et al., 2016). It is possible unmeasured factors aside from treatment group contributed to this result.

Pain behavior was the most challenging of the outcomes to synthesize; not only did the studies measuring these outcomes potentially differ with regard to study design, specifics of treatment groups, and potential complications from extracting data from graphs, but also the duration of observation period differed between studies. The studies included in the pain behavior meta-analyses were the most varied in this respect, and this may be why overall effects were not consistent both within an analysis and between analyses for different outcomes at a similar time point. Overall, it is reasonable to conclude from our findings that some protective effect of NSAID use on pain behavior exists, and that this effect is seen after the duration of the effect of the local anesthetic and mitigates the delayed cortisol rise when no NSAID is given. However, based on the difficulties in combining studies in this area, it is not possible to estimate the exact nature of the effect nor its duration.

\section{Clinical Relevance}

The administration of local anesthetic was beneficial for reduction of the acute pain associated with cautery disbudding, with the caveat that a delayed rise in plasma cortisol occurred after the duration of effect of the local anesthetic. Administration of an NSAID in addition to local anesthetic showed benefits in the hours following the effect of the local anesthetic through reduced plasma cortisol, pain behaviors, and increased pressure sensitivity. Therefore, an NSAID and local anesthetic should be recommended over local anesthetic alone. However, the multitude of different NSAID, routes, dosages, and treatment intervals used in the studies included in the data synthesis likely contribute to much of the heterogeneity seen, driven in part driven by pharmacodynamics differences. The range of both different treatments and outcomes ordained an insufficient number of similar treatments measuring comparable outcomes to combine for subgroup analysis to explore what drove this heterogeneity. This precludes any overall conclusion on which specific NSAID treatment protocol resulted in the best outcomes.

\section{Implications for Future Research}

Both pressure sensitivity and pain behavior may be useful indicators of pain in the hours and days following disbudding; however, more standardization in study design, especially for pain behavior, would allow for 
a greater ability to synthetize results from multiple studies. Whenever possible, total counts for entire time periods should be used to avoid potential bias due to random variation with short sampling intervals.

For all outcomes measured, reporting of numerical data in addition to figures are recommended (Sargeant et al., 2010). With online publication, supporting documents such as tables of all data collected (for example, in the case of outcomes with nonsignificant results the authors' do not, at the time, consider substantial) could be a requirement of the scientific journal to facilitate future data synthesis. The amount, quality, and combinability of data available from primary studies determines the ability to conduct a meta-analysis and influences the precision of the overall effect estimate.

Risk of bias assessment of studies included in our review was challenging, as much important information was often unreported. Studies that fail to report key design features, or those lacking sound methods, may result in biased effect estimates (Sargeant et al., 2010). Scientific journals that do not currently require authors and reviewers to follow reporting guidelines should reconsider their stance. For clinical trials in livestock species, use of The REFLECT Statement (O'Connor et al., 2010; Sargeant et al., 2010) could help better ensure quality information is available to end users.

\section{CONCLUSIONS}

Based on reductions in plasma cortisol, pain behaviors, and pressure sensitivity, we found that the use of local anesthetic and an NSAID is best practice for pain mitigation for cautery disbudding of calves 12 wk of age or less. The magnitude and duration of the effect of NSAID treatment was not possible to deduce from the literature, as much variation existed between studies. It is likely that differences in pharmacodynamic properties of the different NSAID treatments were responsible for much of the heterogeneity; however, there were not enough similar treatments measuring similar outcomes to conduct subgroup analysis to fully explore differences by treatments. This information would be useful to guide more specific veterinary recommendations, and we recommend consideration of more standardized outcome measurements in future research, especially for pain behaviors. In addition, adherence to reporting guidelines by authors would ensure more transparent and complete information available to end users.

\section{ACKNOWLEDGMENTS}

This project was funded by a Knowledge Translation and Transfer grant from the Agri-Food and Rural Link program of the Ontario Ministry of Agriculture, Food, and Rural Affairs - University of Guelph partnership (Guelph, Ontario, Canada).

\section{REFERENCES}

Adams, A. E., J. E. Lombard, C. S. Shivley, N. J. Urie, I. N. RomanMuniz, C. P. Fossler, and C. A. Kopral. 2015. Management practices that may impact dairy heifer welfare on U.S. dairy operations. J. Dairy Sci. 98:105. (Abstr.)

Allen, K. A., J. F. Coetzee, L. N. Edwards-Callaway, H. Glynn, J. Dockweiler, B. KuKanich, H. Lin, C. Wang, E. Fraccaro, M. Jones, and L. Bergamasco. 2013. The effect of timing of oral meloxicam administration on physiological responses in calves after cautery dehorning with local anesthesia. J. Dairy Sci. 96:5194-5205.

American Veterinary Medical Association. 2014. Literature review on the welfare implications of the dehorning and disbudding of cattle. Accessed Mar. 29, 2017. https://www.avma.org/KB/Resources/ LiteratureReviews/Pages/Welfare-Implications-of-Dehorning-and -Disbudding-Cattle.aspx.

Boandl, K. E., J. E. Wohlt, and R. V. Carsia. 1989. Effects of handling, administration of a local anesthetic, and electrical dehorning on plasma cortisol in Holstein calves. J. Dairy Sci. 72:2193-2197.

Canadian Veterinary Medical Association. 2010. Disbudding and dehorning of cattle - Position statement. Accessed Mar. 29, 2017. http://www.canadianveterinarians.net/documents/disbudding -and-dehorning-of-cattle.

Dairy Farmers of Canada. 2015. proAction: Leading the way for sustainable dairy farming; providing assurance to customers about farm practices. Dairy Farmers of Canada, Ottawa, Ontario, Canada. Accessed Mar. 29, 2017. https://www.dairyfarmers.ca/Media/ Files/proaction/proaction_ang_lr15.pdf.

Doherty, T. J., H. G. Kattesh, R. J. Adcock, M. G. Welborn, and A. M. Saxton. 2007. Effects of a concentrated lidocaine solution on the acute phase stress response to dehorning in dairy calves. J. Dairy Sci. 90:4232-4239.

Duffield, T. F., A. Heinrich, S. T. Millman, A. DeHaan, S. James, and K. Lissemore. 2010. Reduction in pain response by combined use of local lidocaine anesthesia and systemic ketoprofen in dairy calves dehorned by heat cauterization. Can. Vet. J. 51:283-288.

Faulkner, P. M., and D. M. Weary. 2000. Reducing pain after dehorning in dairy calves. J. Dairy Sci. 83:2037-2041.

Graf, B., and M. Senn. 1999. Behavioural and physiological responses of calves to dehorning by heat cauterization with or without local anesthesia. Appl. Anim. Behav. Sci. 62:153-171.

Grøndahl-Nielsen, C., H. B. Simonsen, J. Damkjerlund, and M. Hesselholt. 1999. Behavioural, endocrine and cardiac responses in young calves undergoing dehorning without and with use of sedation and analgesia. Vet. J. 158:14-20.

Heinrich, A., T. F. Duffield, K. D. Lissemore, and S. T. Millman. 2010. The effect of meloxicam on behavior and pain sensitivity of dairy calves following cautery dehorning with a local anesthetic. J. Dairy Sci. 93:2450-2457.

Heinrich, A., T. F. Duffield, K. D. Lissemore, E. J. Squires, and S. T. Millman. 2009. The impact of meloxicam on postsurgical stress associated with cautery dehorning. J. Dairy Sci. 92:540-547.

Higgins, J. P., D. G. Altman, P. C. Gotzsch, P. Juni, D. Moher, A. D. Oxman, and J. A. C. Sterne. 2011. The Cochrane Collaboration's tool for assessing risk of bias in randomized trials. BMJ 343:d5928 https://doi.org/10.1136/bmj.d5928.

Huber, J., T. Arnholdt, E. Möstl, C. C. Gelfert, and M. Drillich. 2013 Pain management with flunixin meglumine at dehorning of calves. J. Dairy Sci. 96:132-140.

Korkmaz, M., Z. K. Saritas, A. Bülbül, and I. Demirkan. 2015. Effect of pre-emptive dexketoprofen trometamol on acute cortisol, inflammatory response and oxidative stress to hot-iron disbudding in calves. Kafkas Univ. Vet. Fak. Derg. 21:563-568.

Milligan, B. N., T. Duffield, and K. Lissemore. 2004. The utility of ketoprofen for alleviating pain following dehorning in young dairy calves. Can. Vet. J. 45:140-143. 
Mintline, E. M., M. Stewart, N. R. Cox, G. A. Verkerk, J. M. Stookey, J. R. Webster, and C. R. Tucker. 2013. Play behavior as an indicator of animal welfare: Disbudding in dairy calves. Appl. Anim. Behav. Sci. 144:22-30.

Moher, D., A. Liberati, J. Tetzlaff, D. G. Altman, and The PRISMA Group. 2010. Preferred reporting items for systematic reviews and meta-analyses: The PRISMA statement. Int. J. Surg. 8:336-341.

Moher, D., L. Shamseer, M. Clarke, D. Ghersi, A. Liberati, M. Petticrew, P. Shekelle, L.A. Stewart, and PRISMA-P Group. 2015. Preferred reporting items for systematic review and meta-analysis protocols (PRISMA-P) 2015 statement. Syst. Rev. 4:1-9. PubMed

Morisse, J. P., J. P. Cotte, and D. Huonnic. 1995. Effect of dehorning on behaviour and plasma cortisol responses in young calves. Appl. Anim. Behav. Sci. 43:239-247.

O'Connor, A. M., J. M. Sargeant, I. A. Gardner, J. S. Dickson, M. E. Torrence, C. E. Dewey, I. R. Dohoo, R. B. Evans, J. T. Gray, M. Greiner, G. Keefe, S. L. Lefebvre, P. S. Morley, A. Ramirez, W. Sischo, D. R. Smith, K. Snedeker, J. Sofos, M. P. Ward, and R. Wills. 2010. The REFLECT statement: Methods and processes of creating Reporting Guidelines For Randomized Controlled Trials for livestock and food safety. Prev. Vet. Med. 93:11-18.

Petrie, N. J., D. J. Mellor, K. J. Stafford, R. A. Bruce, and R. N. Ward. 1996. Cortisol responses of calves to two methods of disbudding used with or without local anesthetic. N. Z. Vet. J. 44:9-14.

Rohatgi, A. 2017. WebPlotDigitizer version 3.12. Austin, TX. Accessed Jun. 15, 2017. https://automeris.io/WebPlotDigitizer/.

Sargeant, J. M., and A. M. O'Connor. 2014. Introduction to systematic reviews in animal agriculture and veterinary medicine. Zoonoses Public Health 61:3-9.

Sargeant, J. M., A. M. O'Connor, I. A. Gardner, J. S. Dickson, M. E. Torrence, I. R. Dohoo, S. L. Lefebvre, P. S. Morley, A. Ramirez, and K. Snedeker. 2010. The REFLECT statement: Reporting guidelines for randomized controlled trials in livestock and food safety: Explanation and elaboration. Zoonoses Public Health 57:105-136.

Stilwell, G., R. C. Carvalho, N. Carolino, M. S. Lima, and D. M. Broom. 2010. Effect of hor-iron disbudding on behaviour and plasma cortisol of calves sedated with xylazine. Res. Vet. Sci. $88: 188-193$

Stilwell, G., M. S. Lima, R. C. Carvalho, and D. M. Broom. 2012. Effects of hot-iron disbudding, using regional anesthesia with and without carprofen, on cortisol and behaviour of calves. Res. Vet. Sci. 92:338-341.

Stilwell, G. T. 2009. Pain evaluation and control after routine interventions in cattle. PhD Diss. Dept. of Animal Behavior and Welfare, Universidade Téchnica de Lisboa, Lisbon, Portugal.

Stock, M. L. 2015. Comparison of oral non-steroidal anti-inflammatory drugs in cautery dehorned calves. PhD Diss. Dept. of Biomedical Science, Iowa State University, Ames.

Stock, M. L., S. L. Baldridge, D. Griffin, and J. F. Coetzee. 2013. Bovine dehorning: Assessing pain and providing analgesic management. Vet. Clin. North Am. Food Anim. Pract. 29:103-133.

Stock, M. L., L. A. Barth, N. K. Van Engen, S. T. Millman, R. Gehring, C. Wang, E. A. Voris, L. W. Wulf, L. Labeur, W. H. Hsu, and J. F. Coetzee. 2016. Impact of carprofen administration on stress and nociception responses of calves to cautery dehorning. J. Anim. Sci. 94:542-555.

Stock, M. L., S. T. Millman, L. A. Barth, N. K. Van Engen, W. H. Hsu, C. Wang, R. Gehring, R. L. Parsons, and J. F. Coetzee. 2015. The effects of firocoxib on cautery disbudding pain and stress responses in preweaned dairy calves. J. Dairy Sci. 98:6058-6069.

Vasseur, E., F. Borderas, R. I. Cue, D. Lefebvre, D. Pellerin, J. Rushen, K. M. Wade, and A. M. de Passille. 2010. A survey of dairy calf management practices in Canada that affect animal welfare. J. Dairy Sci. 93:1307-1315.

Ventura, B. A., M. A. G. von Keyserlingk, and D. M. Weary. 2015. Animal welfare concerns and values of stakeholders within the dairy industry. J. Agric. Environ. Ethics 28:109-126.

Viechtbauer, W. 2010. Conducting meta-analyses in R with the metafor package. J. Stat. Softw. 36:1-48.

Winder, C. B., S. J. LeBlanc, D. B. Haley, K. D. Lissemore, M. A Godkin, and T. F. Duffield. 2016. Practices for the disbudding and dehorning of dairy calves by veterinarians and dairy producers in Ontario, Canada. J. Dairy Sci. 99:10161-10173. 